\title{
Response to Anti-PD-1 in Uveal Melanoma Without High-Volume Liver Metastasis
}

\author{
Douglas B. Johnson, MD, MSCla; Riyue Bao, PhDb; Kristin K. Ancell, MDa; Anthony B. Daniels, MD, MSc ${ }^{c}$; \\ Deborah Wallace, MSNa; Jeffrey A. Sosman, MD; and Jason J. Luke, MDb
}

\begin{abstract}
Background: Uveal melanoma (UM) is an uncommon melanoma subtype with poor prognosis. Agents that have transformed the management of cutaneous melanoma have made minimal inroads in UM. Methods: We conducted a single-arm phase II study of pembrolizumab in patients with metastatic UM and performed bioinformatics analyses of publicly available datasets to characterize the activity of anti-PD-1 in this setting and to understand the mutational and immunologic profile of this disease. Results: A total of 5 patients received pembrolizumab in this study. Median overall survival was not reached, and median progression-free survival was 11.0 months. One patient experienced a complete response after one dose and 2 others experienced prolonged stable disease ( $20 \%$ response rate, $60 \%$ clinical benefit rate); 2 additional patients had rapidly progressing disease. Notably, the patients who benefited had either no liver metastases or small-volume disease, whereas patients with rapidly progressing disease had bulky liver involvement. We performed a bioinformatics analysis of The Cancer Genome Atlas for UM and confirmed a low mutation burden and low rates of T-cell inflammation. Note that the lack of T-cell inflammation strongly correlated with MYC pathway overexpression. Conclusions: AntiPD-1-based therapy may cause clinical benefit in metastatic UM, seemingly more often in patients without bulky liver metastases. Lack of mutation burden and T-cell infiltration and MYC overexpression may be factors limiting therapeutic responses.
\end{abstract}

ClinicalTrials.gov identifier: NCT02359851

J Natl Compr Canc Netw 2019;17(2):114-117 doi: $10.6004 /$ jnccn.2018.7070

aDepartment of Medicine, Vanderbilt University Medical Center, Nashville, Tennessee; 'bepartment of Medicine, University of Chicago, Chicago, Illinois; 'Department of Ophthalmology, Vanderbilt University Medical Center, Nashville, Tennessee; and dDepartment of Medicine, Robert H. Lurie Cancer Center and Northwestern University, Chicago, Illinois.
Uveal melanoma (UM) is an uncommon but aggressive melanocytic neoplasm arising from the choroid, ciliary body, or iris. Although radiotherapy (RT) and surgery are effective treatment options for a subset of patients with this disease, up to one-third of UMs will ultimately

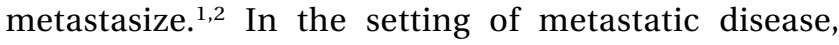
treatment options are limited and prognosis is poor. Immunotherapies, specifically those targeting PD-1 and cytotoxic T-lymphocyte antigen-4 (CTLA-4), have transformed the management of cutaneous melanoma. However, these immunotherapies have demonstrated generally low response rates in retrospective case series, with responses seen in $2 \%$ to $5 \%$ of patients. Thus, effective therapies for this disease remain urgently needed.

Despite the low response rates in retrospective data, anti-PD-1-based therapy has not been explored in a prospective fashion in UM. In addition, retrospective studies may be confounded by poor performance status (PS), hepatic dysfunction, and multiple prior therapies. Thus, the effects of these agents have not been systematically characterized.

To explore this further, we conducted a phase II study of pembrolizumab in metastatic UM. To gain further insights into the correlates of response and resistance, we performed a bioinformatics analysis of The Cancer Genome Atlas (TCGA) for UM.

\section{Methods}

\section{Clinical Trial}

We conducted a multicenter, investigator-initiated phase II study of pembrolizumab in UM from 2015 through 2017 (ClinicalTrials.gov identifier: NCT02359851). Patients with metastatic UM naïve to PD-1-directed agents and with adequate organ function and PS were eligible. To detect a response rate of $20 \%$ (null hypothesis 5\%) using RECIST 1.1, sample size estimates using Simon's optimal design planned for a stage I accrual of 10 patients, with an accrual of an additional 19 patients if one response occurred in the first 10 patients. The study was opened at

\section{See JNCCN.org for supplemental online content.}


A

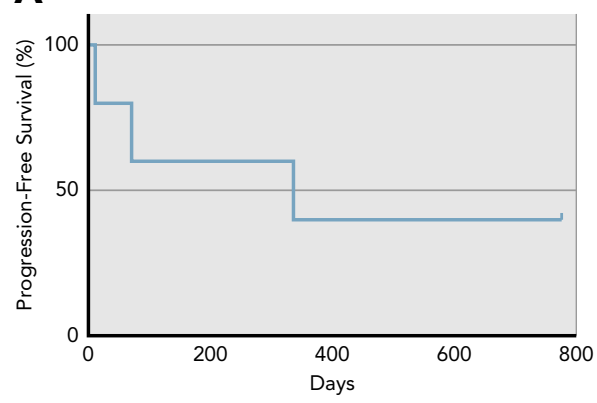

B

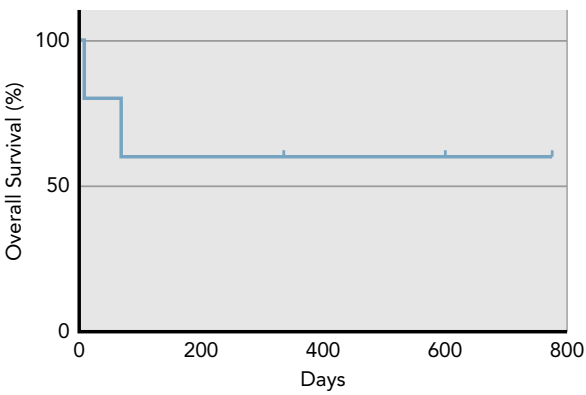

Figure 1. (A) Progression-free survival. (B) Overall survival.

Vanderbilt University Medical Center and University of Chicago.

\section{Bioinformatic Analysis}

The authors queried TCGA for UMs and cutaneous melanomas. ${ }^{5}$ Mutation load was quantified for each sample and compared between UMs and cutaneous melanomas. T-cell inflammation signatures were calculated based on RNA sequencing signatures and were quantified as inflamed, intermediate, and noninflamed as previously published ${ }^{6,7}$; mutation load was then compared between T-cell-inflamed subsets. MYC gene expression and 21 target genes of MYC transcription were quantified in T-cell-inflamed subsets to determine whether MYC signatures correlated with T-cell inflammation.

\section{Results}

We enrolled 5 patients between May 1, 2015, and January 6,2017 . The study was closed early because of slow accrual. Median age was 63 years; 3 patients had liver metastases and 2 had prior therapies (see supplemental eAppendix 1, available online with this article at JNCCN.org). Of these 5 patients, 1 had a complete response (CR; ongoing at 25.5 months), and no partial responses were observed (response rate, 20\%). Another patient experienced prolonged stable disease (ongoing at 11 months), with a decrease in tumor diameter approaching a partial response $(-23 \%)$, and another had stable disease lasting 11 months before experiencing progression (clinical benefit rate $60 \%$ ). The remaining 2 patients experienced rapid progressive disease and functional decline before even obtaining their first CT scan. Note that tumors from patients who benefited were confirmed by molecular sequencing to harbor either GNAQ or GNA11 mutations, but the patients had either no liver involvement or had liver metastases of maximal diameter $<1.2 \mathrm{~cm}$. By contrast, the 2 patients lacking clinical benefit had multiple extensive liver metastases. Median progression-free survival was 11.0 months (Figure 1A) and median overall survival was not reached (Figure 1B; median follow-up, 11.1 months; range, 0.4-25.5 months).
The toxicity profile was consistent with other pembrolizumab studies. The patient who experienced the CR had fulminant type 1 diabetes (grade 4) that arose after the first dose of pembrolizumab, and stopped treatment due to adverse effects and observation of a CR at his first CT scan. Other toxicities among patients included grade 1 hypothyroidism $(n=1)$ and rash $(n=1) ; 3$ patients had no side effects attributed to pembrolizumab. Extremely limited tissue was available for biologic correlates. Two samples, both core biopsies from liver metastases, were able to undergo PD-L1 testing, revealing absence of PD-L1 expression.

During the study, retrospective data became available suggesting an extremely low response rate to PD-1

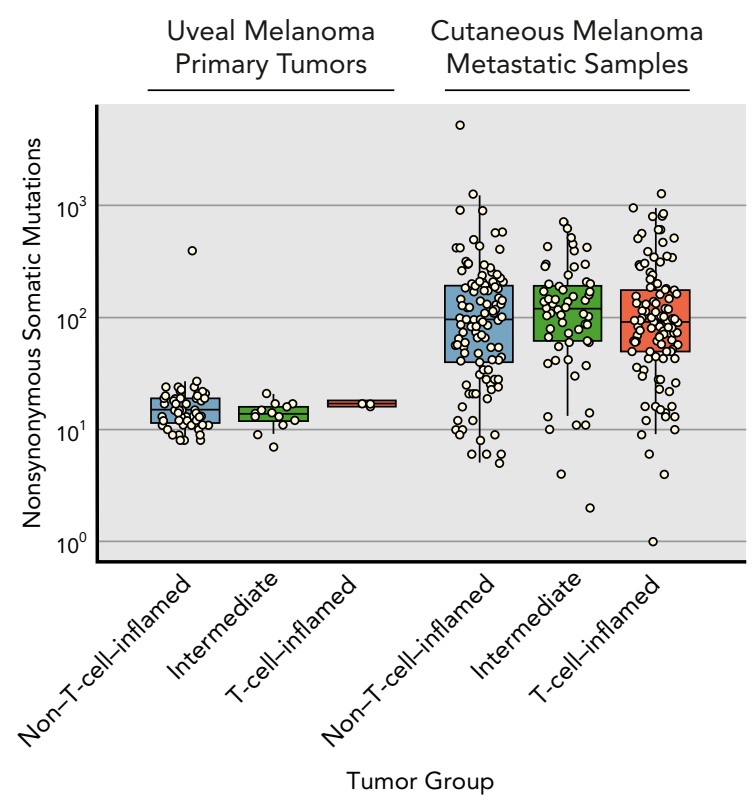

Figure 2. Nonsynonymous tumor mutational load comparing cutaneous metastatic and uveal melanoma. The vertical axis shows the total number of predicted protein-changing somatic mutations (single nucleotide variants and small insertions/deletions). Each data point represents one sample. The horizontal axis shows the non-Tcell-inflamed (blue), intermediate (green), and T-cell-inflamed (red) tumor groups. 


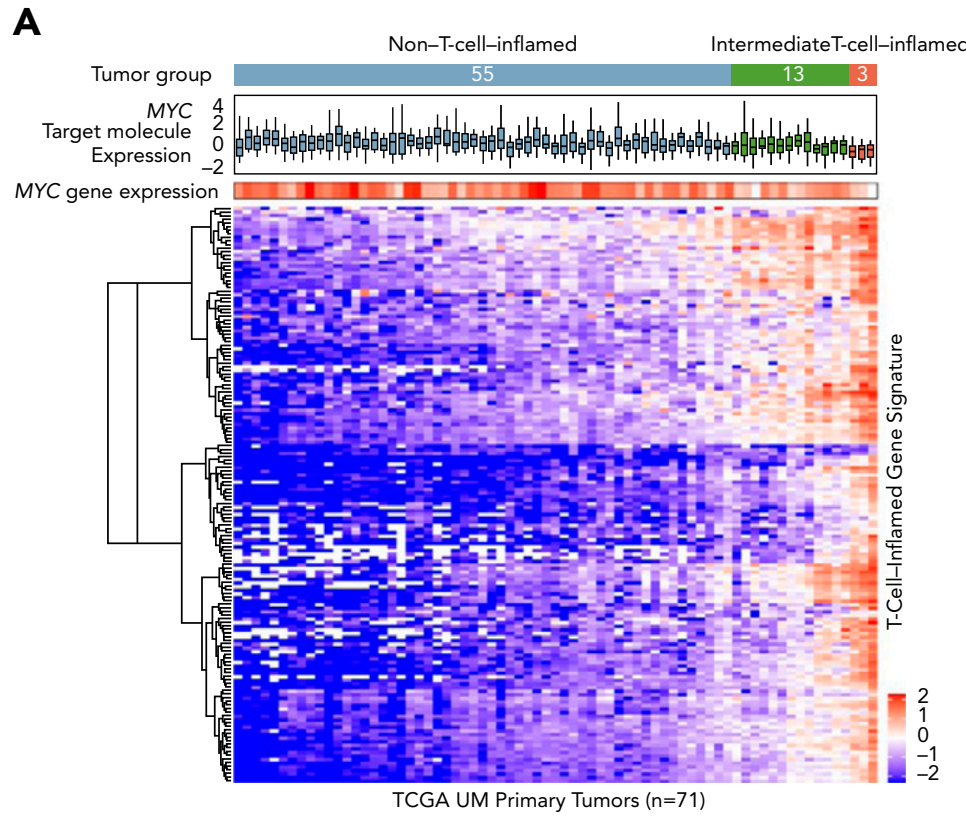

B
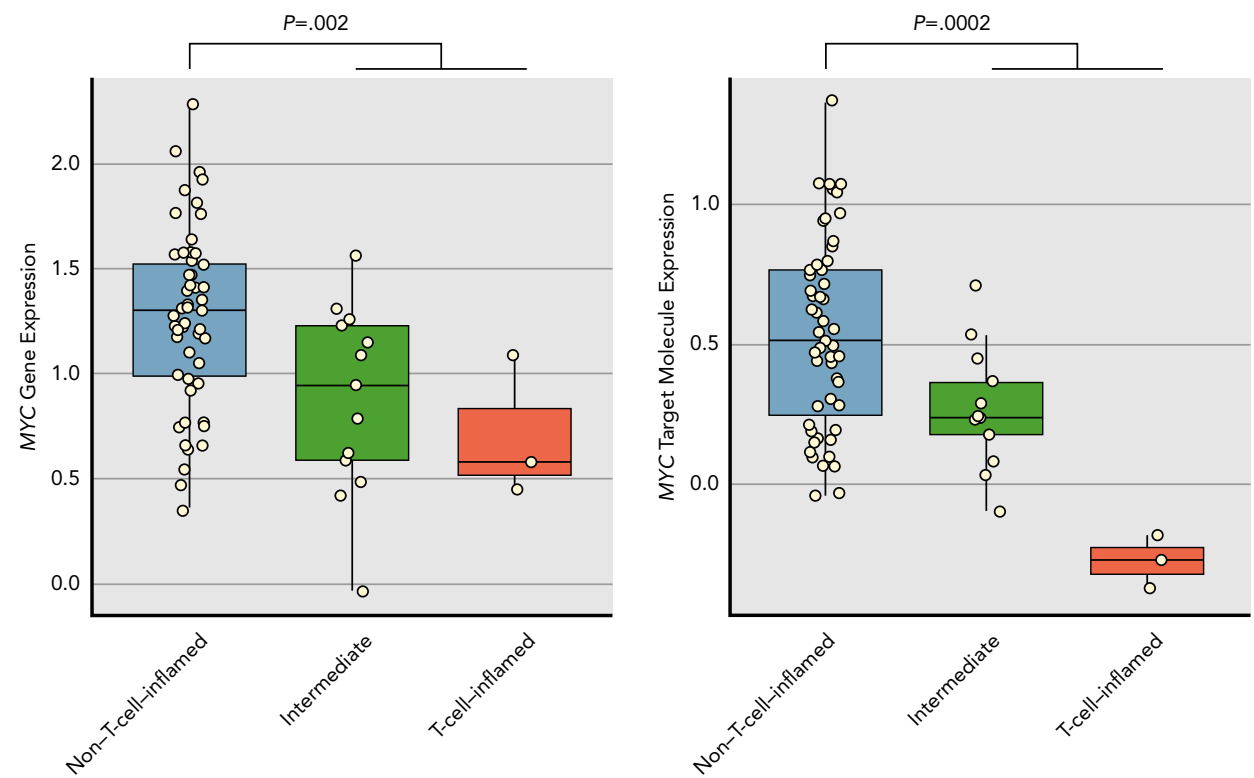

Figure 3. (A) MYC gene and target molecule expression by T-cellinflamed gene signature in UM. Top to bottom: annotation bar showing the non-T-cell-inflamed (blue), intermediate (green), and T-cellinflamed (red) tumor groups of UM samples from TCGA database; boxplot showing the expression distribution of 21 annotated MYC signaling target molecules (CDC25A, CDK2, ECSIT, FASN, FSTL1, FUT3, GGH, HMGA1, IMPA2, IRS1, JARID2, LAMB2, MAG, MYC, MYH7, PYCR1, RTN2, RUVBL1, SLC16A1, SOX9, TKT) defined in Ingenuity Knowledgebase (QIAGEN Inc.) based on experimental evidence small heatmap panel showing the expression pattern of MYC gene; larger heatmap panel showing the expression pattern of T-cell-inflamed gene signature. The heatmap color key appears at the right bottom corner, with blue indicating a lower expression and red indicating a higher expression. (B) MYC gene expression (left) and MYC target molecule expression (right) in the non-T-cell-inflamed (blue), intermediate (green), and T-cell-inflamed (red) tumor groups. $P$ values were computed using 2 -sided Student $t$ test comparing the non-T-cell-inflamed group with the intermediate and T-cell-inflamed groups combined because of limited number of samples in the T-cell-inflamed tumor group. Abbreviations: TCGA, The Cancer Genome Atlas; UM, uveal melanoma. monotherapy. ${ }^{3}$ We were therefore intrigued by the response and clinical benefit observed in our small series. Given the known association of PD-1 response with the density of nonsynonymous somatic mutations $^{8}$ and interferon- $\gamma$-associated gene expression, ${ }^{9}$ we pursued an analysis of TCGA stratifying the uveal melanoma cohort by our previously defined T-cellinflamed tumor microenvironment gene signature (with the caveat that TCGA cancers are largely primary tumors rather than metastases).$^{6}$ Consistent with prior investigations, ${ }^{6,10}$ mutational burden was an order of magnitude lower in UM without a significant difference between T-cell-inflamed and noninflamed tumors (Figure 2). We observed that most of the UMs demonstrated a highly non-T-cell-inflamed tumor microenvironment; however, a small number were T-cell-inflamed (Figures 2 and 3A). In addition, the oncogene $M Y C$, a known mediator of the non-Tcell-inflamed tumor microenvironment, ${ }^{11}$ was significantly upregulated in non-T-cell-inflamed UMs relative to intermediate and T-cell-inflamed UMs, as evidenced by $M Y C$ gene expression $(P=.002)$ and $M Y C$ target molecule expression $(P=.0002$; Figure 3$)$. These data suggest that $M Y C$-directed therapies are 
rational combination approaches with immunotherapy for UM.

\section{Discussion}

This small study demonstrated that pembrolizumab can have activity in metastatic UM. We learned several valuable lessons from this study. First, anti-PD-1 can produce profound and durable clinical benefit for individual patients with UM. Although retrospective data have indicated extremely low response rates, our study suggests that patients with good PS, adequate organ function, and, perhaps most importantly, lack of bulky liver metastases may benefit from therapy. Clearly, however, the small sample size limits generalized conclusions regarding median survival and response rates. Second, accrual to a trial with an approved agent in a rare tumor type is very challenging. Pembrolizumab was approved for cutaneous melanoma in 2014, approximately the same time as our trial's opening. Due to frequent treatment in community practice, exclusion criteria, and subsequent availability of ipilimumab and nivolumab, accrual was a significant challenge, even at institutions that historically have had excellent accrual to UM trials. Finally, our experience corroborates other studies that have suggested that patients with both cutaneous melanoma and UM with bulky liver metastases have poor outcomes and urgently need novel therapeutics. ${ }^{3,12}$ Clinical trials of hepatic-directed therapies in combination checkpoint blockade are underway (ClinicalTrials.gov identifier: NCT02913417). In addition, epigenetic modifier programs targeting $M Y C$ have been proposed ${ }^{13}$ and have begun to be explored in clinical trials (NCT02959437). Consideration should perhaps be given to future clinical trial designs in UM to stratify patients without liver metastases, because they may have substantially better outcomes.

Submitted March 28, 2018; accepted for publication August 6, 2018.

Disclosures: Dr. Johnson has disclosed that he serves on advisory boards for Array BioPharma, Bristol-Myers Squibb, Genoptix, Incyte, Merck, and Novartis and receives research funding from Bristol-Myers Squibb and Incyte. Dr. Luke has disclosed that he consults and receives research funding from Merck. The remaining authors have not received any financial consideration from any person or organization to support the preparation, analysis, results, or discussion of this article.

Funding: Dr. Johnson has disclosed funding from NIH/NCI K23 CA204726 and the James C. Bradford Jr. Melanoma Fund. Dr. Daniels has disclosed funding from NIH/NEI 5K08EY027464-02 and from a Career Development Award from Research to Prevent Blindness. Dr. Luke has disclosed funding from the Department of Defense Career Development Award (W81XWH-16-PRCRPCDA), the Arthur J. Schreiner Family Melanoma Research Fund, the J. Edward Mahoney Foundation Research Fund, and the Brush Family Immunotherapy Fund, and support from the Center for Research Informatics of the University of Chicago Biological Science Division and the Institute for Translational Medicine/CTSA (NIH UL1 RR024999)

Clinical trial funding was provided by Merck (ClinicalTrials.gov identifier: NCT02359851).

Correspondence: Douglas B. Johnson, MD, MSCl, Department of Medicine, Vanderbilt University Medical Center, 777 PRB, 2220 Pierce Avenue, Nashville, TN 37232. Email: Douglas.b.johnson@vanderbilt.edu

\section{References}

1. Diener-West M, Reynolds SM, Agugliaro DJ, et al. Development of metastatic disease after enrollment in the COMS trials for treatment of choroidal melanoma: Collaborative Ocular Melanoma Study Group report no. 26. Arch Ophthalmol 2005;123:1639-1643.

2. Collaborative Ocular Melanoma Study Group. Assessment of metastatic disease status at death in 435 patients with large choroidal melanoma in the Collaborative Ocular Melanoma Study (COMS): COMS report no. 15. Arch Ophthalmol 2001;119:670-676.

3. Algazi AP, Tsai KK, Shoushtari AN, et al. Clinical outcomes in metastatic uveal melanoma treated with PD-1 and PD-L1 antibodies. Cancer 2016; 122:3344-3353.

4. Luke JJ, Callahan MK, Postow MA, et al. Clinical activity of ipilimumab for metastatic uveal melanoma: a retrospective review of the Dana-Farber Cancer Institute, Massachusetts General Hospital, Memorial SloanKettering Cancer Center, and University Hospital of Lausanne experience. Cancer 2013;119:3687-3695.

5. Cancer Genome Atlas Network. Genomic classification of cutaneous melanoma. Cell 2015;161:1681-1696.

6. Spranger S, Luke JJ, Bao R, et al. Density of immunogenic antigens does not explain the presence or absence of the T-cell-inflamed tumor microenvironment in melanoma. Proc Natl Acad Sci U S A 2016;113: E7759-7768.

7. Spranger S, Bao R, Gajewski TF. Melanoma-intrinsic $\beta$-catenin signalling prevents anti-tumour immunity. Nature 2015;523:231-235.

8. Johnson DB, Frampton GM, Rioth MJ, et al. Targeted next generation sequencing identifies markers of response to PD-1 blockade. Cancer Immunol Res 2016;4:959-967.

9. Ayers M, Lunceford J, Nebozhyn M, et al. IFN- $\gamma$-related mRNA profile predicts clinical response to PD-1 blockade. J Clin Invest 2017;127: 2930-2940.

10. Robertson AG, Shih J, Yau C, et al. Integrative analysis identifies four molecular and clinical subsets in uveal melanoma. Cancer Cell 2017;32:204-220.e15.

11. Spranger S, Gajewski TF. Impact of oncogenic pathways on evasion of antitumour immune responses. Nat Rev Cancer 2018;18:139-147.

12. Tumeh PC, Hellmann MD, Hamid $O$, et al. Liver metastasis and treatment outcome with anti-PD-1 monoclonal antibody in patients with melanoma and NSCLC. Cancer Immunol Res 2017;5:417-424.

13. Topper MJ, Vaz M, Chiappinelli KB, et al. Epigenetic therapy ties MYC depletion to reversing immune evasion and treating lung cancer. Cell 2017;171:1284-1300.e21. 